\title{
A high-throughput protocol for isolating cell-free circullating turmor DNA from peripheral blood
}

Pawan K Pandoh', Richard D Corbett', Helen McDonald', Miguel Alcaide ${ }^{3}$, Heather Kirk', Eva Trinh', Simon Haile', Tina MacLeod', Duane Smailus', Steve Bilobram', Andrew J Mungall', Yussanne Ma', Richard A Moore', Robin Coope', Yongjun Zhao', Steven JM Jones ',2,3, Robert A Holt ${ }^{1,2,3}$, Aly Karsan ${ }^{1,4}$, Ryan D Morin ${ }^{1,3}$ \& Marco A Marra ${ }^{\star 1,2}$

\section{ABSTRACT}

The analysis of cell-free circulating tumor DNA (ctDNA) is potentially a less invasive, more dynamic assessment of cancer progression and treatment response than characterizing solid tumor biopsies. Standard isolation methods require separation of plasma by centrifugation, a time-consuming step that complicates automation. To address these limitations, we present an automatable magnetic bead-based ctDNA isolation method that eliminates centrifugation to purify ctDNA directly from peripheral blood (PB). To develop and test our method, ctDNA from cancer patients was purified from PB and plasma. We found that allelic fractions of somatic single-nucleotide variants from target gene capture libraries were comparable, indicating that the PB ctDNA purification method may be a suitable replacement for the plasma-based protocols currently in use.

\section{METHOD SUMMARY}

Circulating cell-free DNA (cfDNA) purification from peripheral blood requires centrifugation to separate plasma from whole blood. We developed a method to purify cfDNA directly from peripheral blood using magnetic beads by modifying binding and lysis conditions. Our method includes a purification step to remove heme and other contaminants known to inhibit PCR amplification.

KEYWORDS

cfDNA - ctDNA - library construction • liquid biopsy $\cdot$ next-generation sequencing - target capture

'Canada's Michael Smith Genome Sciences Centre, BC Cancer, 675 West 10th Avenue, Vancouver, BC, Canada; ${ }^{2}$ Department of Medical Genetics, Faculty of Medicine, University of British Columbia, Vancouver, Canada; ${ }^{3}$ Department of Molecular Biology \& Biochemistry, Simon Fraser University, Burnaby, BC, Canada; ${ }^{4}$ Department of Pathology \& Laboratory Medicine, University of British Columbia, Vancouver, Canada; *Author for correspondence: mmarra@bcgsc.ca

BioTechniques 66:85-92 (February 2019) 10.2144/ btn-2018-0148

\section{INTRODUCTION}

Liquid biopsies, followed by DNA purification and sequence analysis, have the potential to improve cancer detection, non-invasive tumor genotyping, and disease monitoring [1]. Purifications of ctDNA, tumor-derived cfDNA (cell-free DNA), can yield DNA fragments that vary in size. Smaller fragments can be produced by cells undergoing apoptosis, while larger fragments can be produced from necrotic cells [2]. A study by Raymond and colleagues reported that the majority of cfDNA is found in nucleosomal fragments and the expected yield per milliliter of blood is usually below $10 \mathrm{ng}$, averages $6.6 \mathrm{ng}$, and depends on type and stage of tumor [2]. Other factors such as pregnancy, intense exercise, cardiovascular disease and post-radiation cellular damage can affect the abundance of cfDNA yield [2].

cfDNA-based liquid biopsy nextgeneration sequencing (NGS) tests have been developed to interrogate tumor specific somatic alterations $[3,4]$. These tests have been introduced in research and clinical labs at scale, and the need for rapid and high-throughput purification of cfDNA has become apparent. Several methods for isolating cfDNA have been described in the literature [2]. A recent comprehensive review published by Gorgannezhad et al. outlined opportunities, challenges and advances made in the field of ctDNA research [5]. In particular, Gorgannezhad et al. highlighted the need to have standardized methods for collection and storage of PB samples. Schmidt et al. showed that the yield of isolated cfDNA can be significantly increased by using salting-out purification methods instead of column-based methods [6]. They also showed that the separation of plasma from blood requires centrifugation at high speed, causing ex vivo release of
DNA from blood cells [6]. The centrifugation is not only a labor intensive low throughput process, but also is prone to sample contamination and sample swaps.

A commonly used column-based method to isolate cfDNA from plasma and serum is Qiagen's QIAamp circulating nucleic acid kit (Qiagen, Hilden, Germany) [7,8]. The Qiagen cfDNA method involves initial plasma separation using centrifugation and subsequent column-based extraction. To develop a method that is amenable to high-throughput cfDNA purification, we evaluated the magnetic bead-based cfDNA extraction protocol used in the NextPrep-Mag'" cfDNA Isolation Kit, cat\#3825-01 (Bioo Scientific, PerkinElmer, USA). The NextPrep-Mag cfDNA purification kit includes readyto-use reagents and offers a relatively rapid turnaround time of approximately $2 \mathrm{~h}$. We compared the NextPrepMag against Qiagen's QIAamp cfDNA isolation method, our validated in-house production protocol. Although we found that the NextPrep-Mag protocol performed well in terms of nucleic acid yield and quality, the centrifugationbased separation of plasma from whole blood remained a rate-limiting step. Here we describe a magnetic beadbased method we call 'blood-direct' cfDNA isolation. Unlike other beadbased methods that require plasma separation, our blood-direct approach does not require plasma separation or the use of columns and can fit in Society of Biomolecular Screening (SBS) format plates for automation on a liquid handling robot.

\section{MATERIALS \& METHODS}

Patients were consented for our ctDNA study as part of the BC Cancer Agency's Personalized Onco-Genomics (POG) 


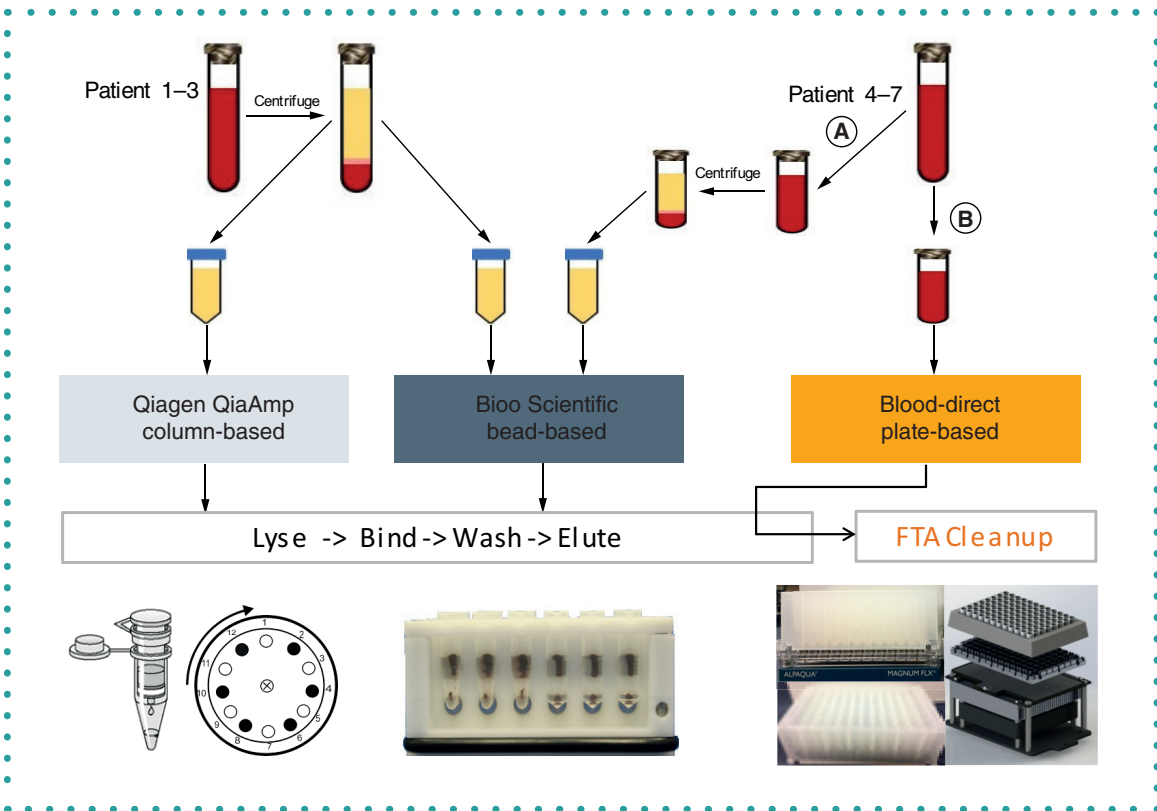

Figure 1. Experimental strategy. $10 \mathrm{ml}$ of peripheral blood were collected from each patient into a Streck tube. For the first three patients, the Qiagen cfDNA isolation method was directly compared with the Bioo Scientific method by equally splitting the plasma after centrifugation. The Qiagen method employs filter cartridges and centrifugation while the Bioo Scientific method requires a magnetic rack to isolate the cfDNA. The Blood-direct (B) and Bioo Scientific methods (A) were evaluated for patients 4-7. The blood-direct cfDNA isolation method does not require centrifugation to separate the plasma and is performed in SBS format labware making it automation friendly.

program [9-13]. $10 \mathrm{ml}$ of peripheral blood from seven cancer patients were collected in Streck tubes, cat\# 218997 (Streck, NB, USA). Three samples were centrifuged for $10 \mathrm{~min}$ at $2000 \times \mathrm{g}$ to separate the plasma, which was then split into two to allow comparison of Qiagen's QIAamp ${ }^{\circledR}$ Circulating Nucleic Acid kit, cat\# 55114, and Bioo Scientific's NextPrep-Mag cfDNA Isolation Kit, cat\#3825-01 (Bioo Scientific), see Figure 1.

NextPrep-Mag cfDNA isolation protocol was also compared to our blood-direct cfDNA isolation approach as follows. $10 \mathrm{ml}$ of peripheral blood (PB) from the remaining patients was split into $A$ and $B$ tubes. Each patient's Tube A was centrifuged as described above to separate plasma and tube $B$ was processed as whole $\mathrm{PB}$ for extracting cfDNA (Table 1 and Figure 1). Briefly, in the blood-direct method, PB was treated with lysis-binding buffer and proteinase $\mathrm{K}$ under denaturing conditions at $55^{\circ} \mathrm{C}$ for $15 \mathrm{~min}$ in the presence of magnetic beads. Following the heating step, the mixture was incubated for $1 \mathrm{~h}$ at room temperature with mixing every 10 min at 1200 rpm for 30 s on an Eppendorf thermomixer. Beads were captured on a magnetic stand for 2 min and then washed three times after which cfDNA was eluted by adding elution buffer and incubating for $5 \mathrm{~min}$ at $55^{\circ} \mathrm{C}$. cfDNA was further purified by diluting in 1:1 FTA (Fast Technology for Analysis) reagent, cat\# WHAWB120204 (Sigma-Aldrich, USA), containing $\mathrm{NaCl}$ (sodium chloride); Tris; EDTA (ethylenediaminetetraacetic acid); TRITON-X-100 (t-Octylphenoxypolyethoxyethanol) and incubated for $10 \mathrm{~min}$ at room temperature. An additional bead purification step was performed using PCRClean DX beads, cat\# C-1003-450 (ALINE Biosciences, USA). A detailed protocol is included in the Supplementary Materials.

An equal proportion of the total DNA yield was used to construct libraries from plasma and whole PB DNA for each paired sample. Libraries were prepared for sequence analysis using master mix reagents, custom catalogue\# E6875B-GSC, E6876B-GSC and E6877B-GSC (New England Biolabs Ltd, USA). cfDNA extracts were directly end-repaired, then bead purified with magnetic beads (Aline Biosciences, USA). The repaired DNA fragments were subsequently adenylated, then ligated with barcoded adapters in an overnight reaction

\section{Library preparation}

at $16^{\circ} \mathrm{C}$ as described by Alcaide et al. [14]. The ligation products were purified using Aline magnetic beads with a 0.8:1 ratio of beads to sample, and amplified in an eightcycle PCR reaction with Phusion Hot Start High Fidelity DNA Polymerase (Fisher Scientific Ltd, Canada) and dual index primers [14]. Library PCR products were purified using beads with a 0.8:1 ratio and analyzed using Quant-iT and caliper high sensitivity DNA assays. An additional bead purification step of libraries pooled for capture removed the remaining adapter products and also reduced the volume of the library pool for the lyophilization step.

\section{Targeted enrichment using IDT xGen Lockdown probes}

All of the 14 constructed ctDNA libraries were pooled and subjected to hybridization capture using a 45 gene panel spanning 144 kb (Supplementary Table 1 for gene names and Supplementary Table 2 for gene coordinates). The hybridization capture reaction was carried out according to the protocol of Alcaide et al. using xGen Universal Blocker-TS Mix (Integrated DNA Technologies, USA) as the blocking reagent [14]. The captured products were amplified with 13 cycles of PCR, and bead purified with a 1:1 volume ratio of capture DNA to beads. The capture library products were assessed using Qubit Fluorometer (ThermoFisher Scientific, Canada) and Agilent Bioanalyzer High Sensitivity DNA assays (Agilent Technologies, Inc., USA).

\section{Sequencing \& bioinformatics analysis}

The target capture library pool was sequenced on HiSeq2500 instruments (Illumina, CA, USA) using V4 chemistry and paired-end 125 base reads following the manufacturer's protocols. Sequenced reads were aligned and analyzed with ProDuSe 0.2 .2 to create BAM files and putative variant calls [14].

Sequencing data from the plasma cfDNA extraction kits were compared by querying for nucleosome sized peaks in the fragment size distributions of the reference-aligned reads. Size distributions were estimated from the outer-distance of paired-read alignments. Only reads with the expected pair orientation were included.

Each patient in this study previously had their metastatic tumor and PB genomes 


\begin{tabular}{|c|c|c|c|c|c|c|c|}
\hline \multicolumn{2}{|c|}{ Sample description } & \multicolumn{2}{|c|}{ Extraction } & \multicolumn{4}{|c|}{ Library } \\
\hline Patient ID & Pathology & $\begin{array}{l}\text { Extraction } \\
\text { method }\end{array}$ & $\begin{array}{l}\text { Extraction } \\
\text { yield/ml blood } \\
\text { (ng) }\end{array}$ & $\begin{array}{l}\text { Library input } \\
\text { (ng) }\end{array}$ & $\begin{array}{l}\text { Total Library } \\
\text { yield (ng) }\end{array}$ & $\begin{array}{l}\text { \% adapter } \\
\text { region }\end{array}$ & $\begin{array}{l}\text { Calculated library } \\
\text { yield }(250- \\
1000 \text { bp) (ng) }\end{array}$ \\
\hline \multirow[t]{2}{*}{ Patient 1} & \multirow{2}{*}{$\begin{array}{l}\text { Pancreas leio- } \\
\text { myosarcoma }\end{array}$} & Qiagen & 17.8 & 17.8 & 441.4 & 1.6 & 434.4 \\
\hline & & Bioo Scientific & 33.9 & 20.0 & 293.2 & 5.1 & 278.3 \\
\hline \multirow[t]{2}{*}{ Patient 2} & \multirow{2}{*}{$\begin{array}{l}\text { Liver cholan- } \\
\text { giocarcinoma }\end{array}$} & Qiagen & 48.0 & 20.0 & 402.3 & 1.0 & 398.3 \\
\hline & & Bioo Scientific & 37.0 & 20.0 & 317.0 & 4.2 & 303.4 \\
\hline \multirow[t]{2}{*}{ Patient 3} & \multirow{2}{*}{$\begin{array}{l}\text { Unknown tis- } \\
\text { sue carcino- } \\
\text { ma-gastric }\end{array}$} & Qiagen & 22.8 & 20.0 & 264.3 & 15.1 & 224.4 \\
\hline & & Bioo Scientific & 19.5 & 19.5 & 200.9 & 51.1 & 98.2 \\
\hline \multirow[t]{2}{*}{ Patient 4} & \multirow{2}{*}{$\begin{array}{l}\text { Lung adeno- } \\
\text { carcinoma }\end{array}$} & Bioo Scientific & 2.7 & 14.8 & 297.8 & 3.8 & 286.6 \\
\hline & & $\begin{array}{l}\text { Blood-direct } \\
\text { ctDNA }\end{array}$ & 8.2 & 29.0 & 226.3 & 9.1 & 205.6 \\
\hline \multirow[t]{2}{*}{ Patient 5} & \multirow{2}{*}{$\begin{array}{l}\text { Unknown tis- } \\
\text { sue carcino- } \\
\text { ma-endocrine }\end{array}$} & Bioo Scientific & 3.6 & 9.0 & 291.5 & 9.2 & 264.7 \\
\hline & & $\begin{array}{l}\text { Blood-direct } \\
\text { ctDNA }\end{array}$ & 13.0 & 45.5 & 66.3 & 81.5 & 12.3 \\
\hline \multirow[t]{2}{*}{ Patient 6} & \multirow{2}{*}{$\begin{array}{l}\text { Liver adeno- } \\
\text { carcinoma }\end{array}$} & Bioo Scientific & 25.4 & 20.0 & 278.7 & 6.4 & 261.0 \\
\hline & & $\begin{array}{l}\text { Blood-direct } \\
\text { ctDNA }\end{array}$ & 29.3 & 20.3 & 381.4 & 3.8 & 366.8 \\
\hline \multirow[t]{2}{*}{ Patient 7} & \multirow{2}{*}{$\begin{array}{l}\text { Large intes- } \\
\text { tine-colon- } \\
\text { colorectal } \\
\text { cancer }\end{array}$} & Bioo Scientific & 3.16 & 7.9 & 187.4 & 13.2 & 162.7 \\
\hline & & $\begin{array}{l}\text { Blood-direct } \\
\text { ctDNA }\end{array}$ & 9.0 & 31.5 & 106.3 & 26.7 & 77.9 \\
\hline $\begin{array}{l}\text { cfDNA yields } \\
\text { removing the } \\
\text { in the electro }\end{array}$ & $\begin{array}{l}\text { s and subsequen } \\
\text { e adapter peak. } N \\
\text { opherogram. cfD }\end{array}$ & $\begin{array}{l}\text { Ilumina library yi } \\
\text { te that some libra }\end{array}$ & ds are compared & across three ext & ction methods & umina library & $\begin{array}{l}\text { was calculated after } \\
\text { area under the curve }\end{array}$ \\
\hline
\end{tabular}

sequenced to $80 \mathrm{X}$ and $40 \mathrm{X}$, respectively, which yielded somatic variants overlapping the capture regions targeted in this ctDNA experiment. These previously generated genome sequence data were aligned to $\mathrm{hg} 19$ using BWA mem 0.7.6a [15], duplicates were marked with Sambamba [16] and somatic variant calls in the tumor were identified using Strelka [17] to create a set of somatic variants that we examined in the ctDNA sequence data.

Allelic fractions were collected in the ctDNA data by dividing the number of non-duplicate aligned reads containing the non-reference allele by the total number of non-duplicate reads covering that position. For the plasma extraction comparison, three patient samples contained five total somatic variants overlapping the ctDNA capture space. At each of these five positions, the observed allelic fraction was compared between the metastatic genome and the two plasma ctDNA methods and reported in Table 2. Similarly, in the plasma and blooddirect comparison, there were 4 patient samples containing 19 genome-derived somatic variants overlapping with the capture space. Raw allele counts for these data are available in Table 2.

\section{RESULTS \& DISCUSSION}

cfDNA fragment lengths can vary, with typical cfDNA profiles showing an approximately 165 bp chromatosome (145 nucleosome +20 bp linker histone $\mathrm{H} 1$ ) and $330 \mathrm{bp}$ dimer as the dominant DNA species where tumor-specific mutations are routinely observed $[4,18]$. cfDNA derived from PB or plasma had similar size profiles (Figure 2A $\& B$, which encouraged us to proceed with library construction, target capture and sequencing experiments. Since library construction did not include a DNA fragmentation step, larger fragments were unlikely to make it to the final library product. Furthermore, the targeted ctDNA capture method, using $120 \mathrm{nt}$ biotinylated oligonucleotides as baits for capture, has the advantage of reducing larger genomic DNA fragments from the final library pool. The ideal library insert size for target capture reported in the literature is below $300 \mathrm{bp}$ [19]. For the aforementioned reasons, we chose not to perform an upper-bound size selection to remove larger library fragments, as bead-based size selection leads to overall loss in library yield and therefore library complexity. Genomic DNA contamination in the cfDNA preparation is a concern for any preparation method as it dilutes down the mutant DNA alleles. High molecular weight genomic DNA traces were not visible in our Agilent profiles and the extraction yields and profiles were comparable to plasma-based techniques. Furthermore, we were able to detect low frequency allelic variants in our sequencing 


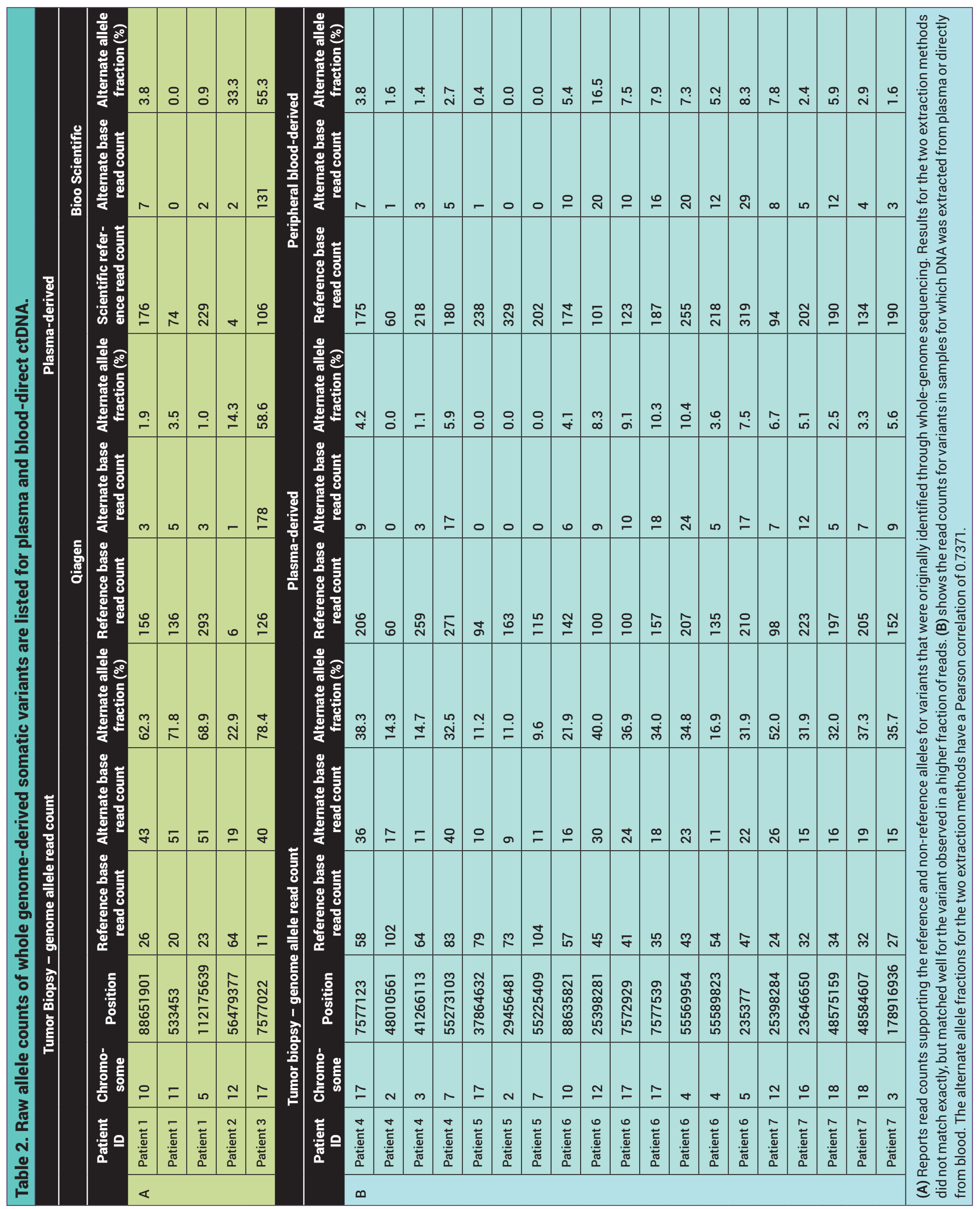


$\checkmark$ data, compatible with the notion that we did not have significant genomic DNA present in our cfDNA preparation.

We noted that cfDNA extracted from whole $P B$ retained a brownish green color and Agilent Bioanalyzer traces appeared dissimilar in comparison to the profiles generated from plasma separated cfDNA. cfDNA concentrations in the blood-direct extraction method were significantly higher than those obtained using the plasma derived method (Figure 3). Peripheral blood is a complex mixture and hence contaminants in cfDNA preparation could have interfered with the fluorescence dye present in the Agilent High Sensitivity and Qubit fluorometeric assays. We hypothesized that heme compounds present in whole blood but not in plasma were causing interference in the electropherograms, and also resulting in the brownish green tint in the blood-direct derived cfDNA. Heme is a known inhibitor of enzymatic reactions, particularly PCR [20], so we performed a purification using FTA purification reagent, containing EDTA as chelating agent, to remove heme and other potential contaminants from $\mathrm{PB}$ derived cfDNA. FTA reagent has been used successfully to isolate nucleic acids from dried blood spots collected on Whatman FTA cards (Sigma-Aldrich, USA) [21,22]. Figure 4 shows size profiles of cfDNA extracted before and after FTA clean-up. Final library construction yield for blooddirect cfDNA was, on average, lower than plasma derived samples (Table 1). This supported the hypothesis that our blooddirect cfDNA prep had contaminants affecting the Qubit quantitation (Figure 2B). Library size profiles from cfDNA extracted directly from whole blood were similar to those extracted from plasma (Figure 2B). Figure 5 shows the estimated fragment size distributions of all three methods where all libraries showed enrichment for nucleosome-sized peaks.

We assessed reproducibility of blooddirect cfDNA isolation by comparing the DNA yield with that of Bioo Scientific's NextPrep-Mag cfDNA isolation method for 81 patient samples and found a higher yield in the former $(p=0.022$, paired two-tailed t-test) (Figure 6). We also assessed the stability of cfDNA by assessing yield change vs. time using Qubit fluorometer

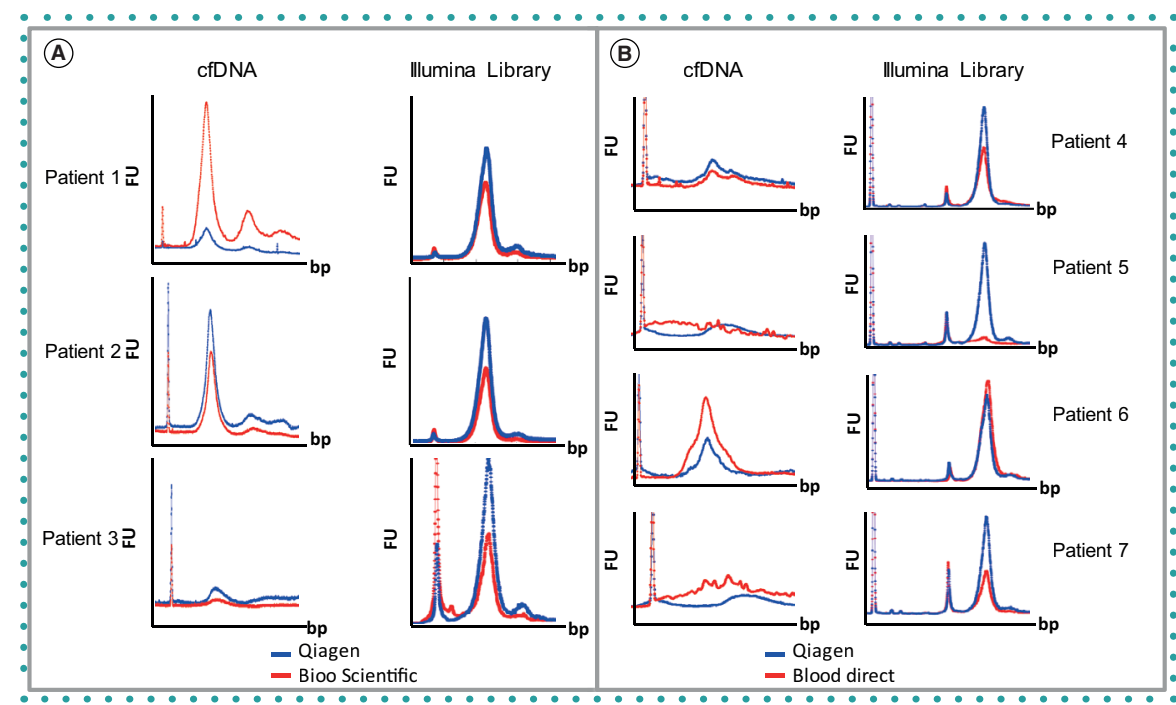

Figure 2. Size profiles of cfDNA purifications and Illumina libraries constructed from them. (A) Agilent bioanalyzer traces comparing extraction methods and final library profiles. cfDNA profiles are variable between samples. The Bioo Scientific and Qiagen methods performed equivalently. The library profiles indicated that both Qiagen and Bioo Scientific extracted cfDNA were suitable templates for Illumina library construction. (B) Agilent bioanalyzer traces comparing extraction methods and final library profiles. Blood-direct derived cfDNA profiles are noisier but clearly show typical cfDNA hallmarks. The lack of discernible differences in the Illumina library profiles indicates that blood-direct cfDNA can be a suitable template for library preparation.

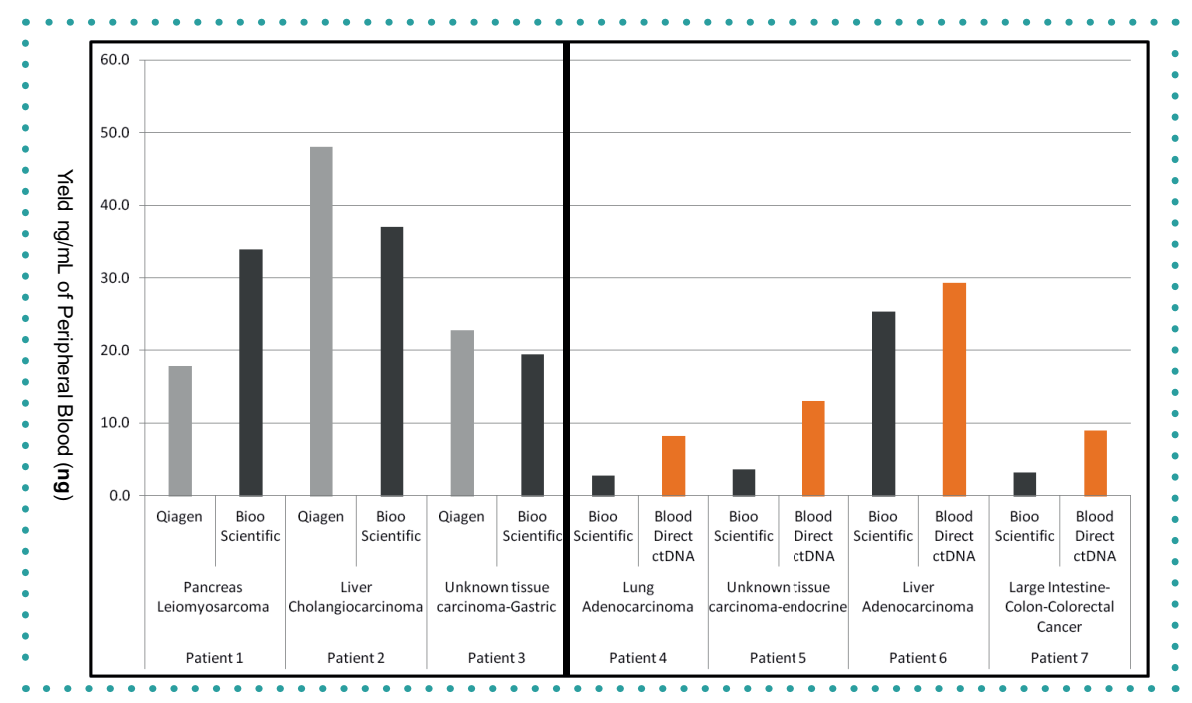

Figure 3. Comparing cfDNA yield by extraction method. Peripheral blood from patients 1-3 was used to validate Bioo Scientific's NextPrep-Mag kits against the Qiagen's QiaAmp circulating nucleic acid extraction method. cfDNA extracted from patients 4-7 peripheral blood was used to evaluate our blood-direct method against Bioo Scientific's NextPrep-mag method.

(Figure 7) and Agilent Bioanalyzer (Figure 8). There was no significant change in cfDNA yield, over a period of 13.1 months. Moreover, before and after Agilent Bioanlyzer traces (Figure 8) were compared and showed no change in specific nucleosomal peaks over time, providing some assurance that cfDNA remains stable while stored at proper temperature and in optimal buffer conditions.
Using results from the ProDuSealigned reads [14] we compared the allelic fractions of 19 genome-sequence derived somatic single-nucleotide variants (SNVs) across four cases with matched blood-direct and plasma ctDNA data. Of the 19 SNVs, 15 were found in the plasma ctDNA and 16 were found in the blood-direct using a cutoff of $0.5 \%$ allelic ratio. It is difficult to attribute the one 


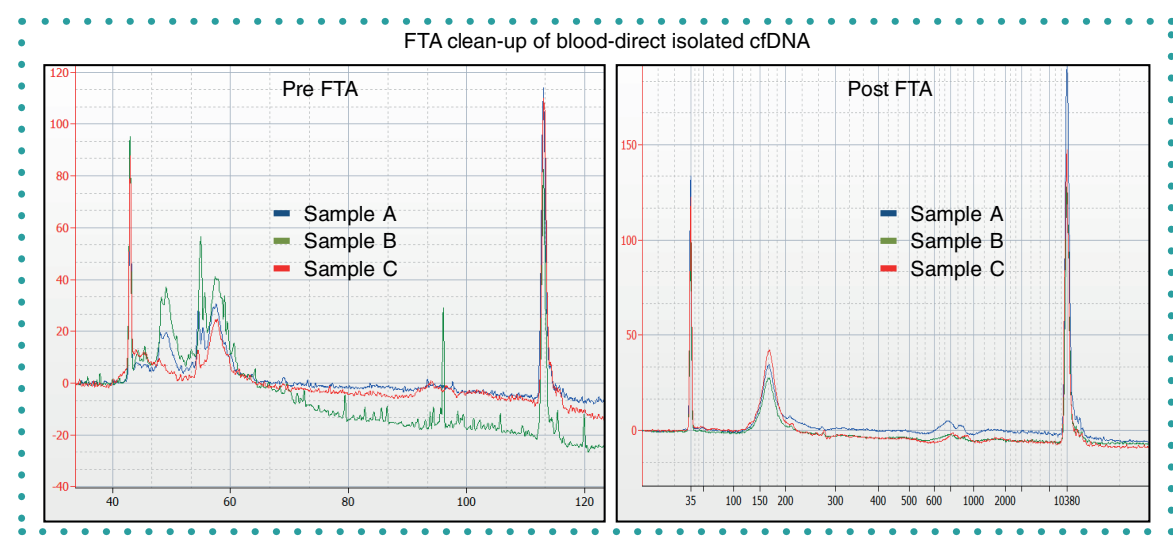

Figure 4. FTA reagent effectively removed contaminants causing aberrant Agilent traces.

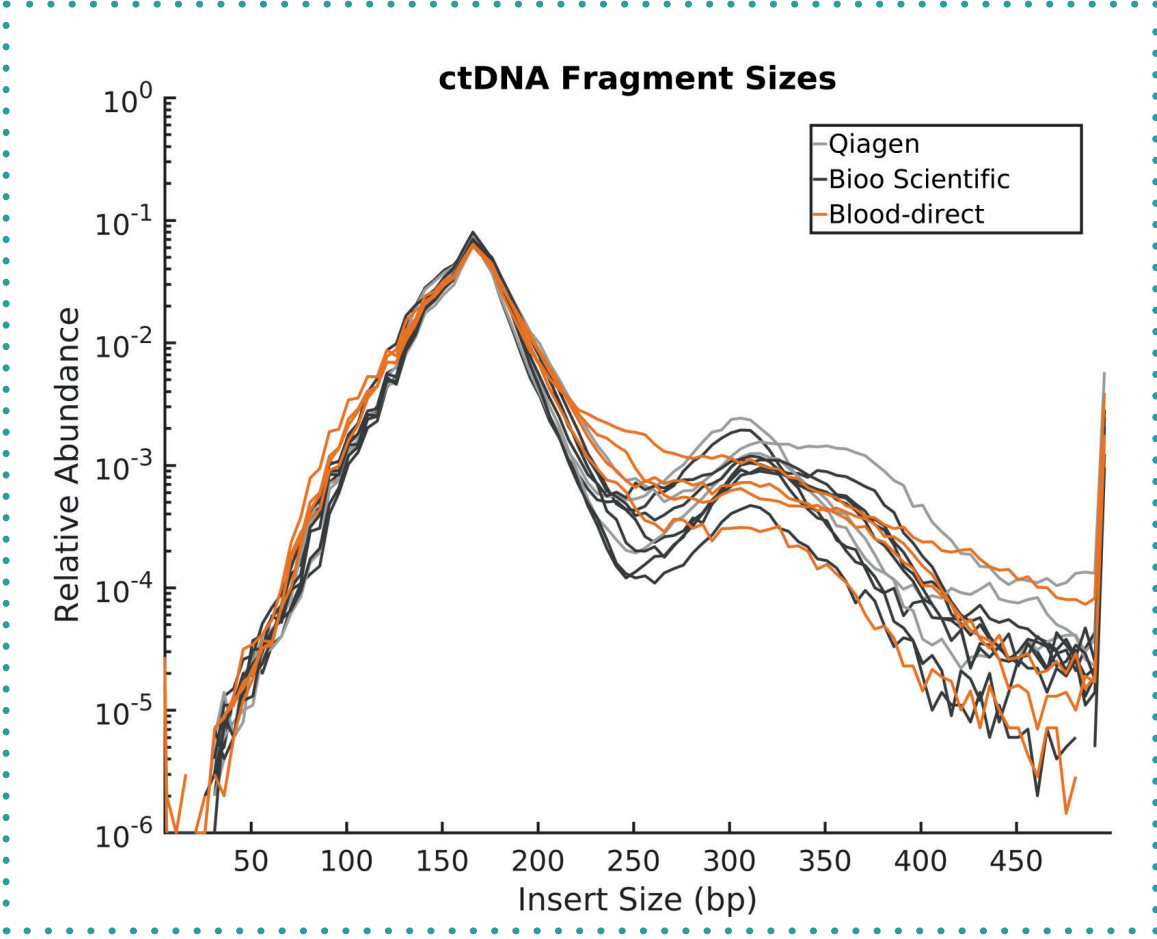

Figure 5. Comparison of cfDNA fragment size distributions from plasma and the blood-direct method. Size distributions of the plasma extracted DNA look comparable to the blood-direct cfDNA extraction method. Peaks are expected in multiples of $145 \mathrm{bp}$ due to cfDNA presence in nucleosomes.

$\checkmark$ difference to anything of biological interest as it had only a single read of support in the blood-direct library (see Table 2) and could possibly be due to sequencing error. Importantly, a strong correlation of the SNV allelic fractions in the gene panel target space was observed between the matching plasma and blood ctDNA samples (four pairs, 19 total SNVs; Pearson's rho $=0.7371$, Figure 9).

The similarity of allele presence in the plasma and blood derived ctDNA libraries, in concert with the expected fragment size distribution has led us to conclude that isolation directly from blood, making the entire work flow automation friendly and thus amenable to higher throughput.

\section{FUTURE PERSPECTIVE}

cfDNA analysis has already seen significant uptake for non-invasive prenatal testing and there are tremendous efforts to do the same for cancer research and care. There thus exists a large and growing need to purify cfDNA at a large scale, with minimal handling and therefore reduced chance of laboratory error. The blood-direct magnetic bead based approach is both automation friendly and suitable for low-throughput, manual handling for field or point of care applications, where lab equipment may not be available. This ability to operate across a range of scales, from large to small, enables several types of analysis. We can envision cfDNA being analyzed in the clinic in the contexts of e.g., cancer, kidney disease and transplant rejection. Coupled with third-generation sequencing technologies, such as from Oxford Nanopore, cfDNA analyses have the potential to offer simple, fast and cost-effective solutions to not only assay for low allelic ratio mutations but also methylation status and other features of the epigenome.

\section{SUPPLEMENTARY DATA}

To view the supplementary data that accompany this paper please visit the journal website at: www.future-science. com/doi/suppl/10.2144/btn-2018-0148

\section{AUTHOR CONTRIBUTIONS}

$\mathrm{PKP}, \mathrm{YZ}$ and $\mathrm{RC}$ conceived and designed the experiments. PKP, HM, ET and HEK performed the experiments. RDC, PKP and YM analyzed the data. PKP and MAM prepared the manuscript. MAM and $Y Z$ supervised the study. MAM, TM, SH, RM, DS, $\mathrm{SJ}, \mathrm{RH}$ and $\mathrm{AK}$ reviewed and corrected the manuscript.

\section{ACKNOWLEDGMENTS}

The authors gratefully acknowledge the support and contributions of the Genome Sciences Centre management and administration group, including the projects team, finance team, lab operations team, and especially Robyn Roscoe, Diane Miller, Katherine Mui, Payal Sipahimalani and Mahsa Amirabbasi. 


\section{FINANCIAL \& COMPETING INTERESTS DISCLOSURE}

This work was supported by funding provided by Genome Canada, Genome BC, Canadian Institutes for Health Research (CIHR) and NSERC - Collaborative Health Research Project, BC Cancer Foundation, Canada Foundation for Innovation/BC Knowledge Development Fund and a CIHR Award to MAM (\#FDN-143288). The authors have no other relevant affiliations or financial involvement with any organization or entity with a financial interest in or financial conflict with the subject matter or materials discussed in the manuscript apart from those disclosed.

No writing assistance was utilized in the production of this manuscript.

\section{ETHICAL DISCLOSURE}

The authors state that they have obtained appropriate institutional review board approval to conduct this study. In addition, informed consent was obtained from the participants involved.

\section{OPEN ACCESS}

This work is licensed under the AttributionNonCommercial-NoDerivatives 4.0 Unported License. To view a copy of this license, visit http://creativecommons.org/licenses/ by-nc-nd/4.0/

\section{REFERENCES}

1. Bettegowda C, Sausen M, Leary RJ et al. Detection of circulating tumor dna in early- and late-stage human malignancies. Sci. Transl. Med. 6(224), ra24 (2014).

2. Volckmar AL, Sultman $\mathrm{H}$, Riediger $\mathrm{A}$ et al. A field guide for cancer diagnostics using cell-free DNA: From principles to practice and clinical applications. Gene principles to practice and clinical applicatio
Chromosome Canc. 57, 123-139 (2018).

3. Pereira E, Camacho-Vanegas $\mathrm{O}$, Anand S et al. Personalized circulating tumor dna biomarkers dynamically predict treatment response and survival in gynecologic cancers. PLoS One 10(12), e0145754 (2015).

4. Murtaza M, Dawson S, Pogrebniak K et al. Multifocal clonal evolution characterized using circulating tumor DNA in a case of metastatic breast cancer. Nat. Commun. 6(8760), (2015).

5. Gorgannezhad L, Umer M, Islam N, Nguyen NT, Muhammad JA, Shiddiky MJA. Circulating tumor DNA and liquid biopsy: opportunities, challenges, and recen advances in detection technologies. Lab Chip. 18(8), 1174-1196 (2018).

6. Schmidt B, Weickmann S, Witt C, Fleischhacker M. Improved method for isolating cell-free DNA. Clin. chem. 51(8), 1561-1563 (2005).

7. Raymond CK, Hernandez J, Karr R, Hill K, Li M. Collection of cell-free DNA for genomic analysis of solid tumors in a clinical laboratory setting. PLoS One 12(4), e0176241 (2017)

8. Scherer F, Kurtz DM, Newman AM et al. Distinct biological subtypes and patterns of genome evolution in lymphoma revealed by circulating tumor DNA. Sci. Transl. Med. 8(364), ra155 (2016).

9. Chahal M, Pleasance E, Grewal J et al. Personalized oncogenomic analysis of metastatic adenoid cystic carcinoma: using whole-genome sequencing to inform clinical decision-making. Cold Spring Harb. Mol. Case Stud. 4(2), a002626 (2018).

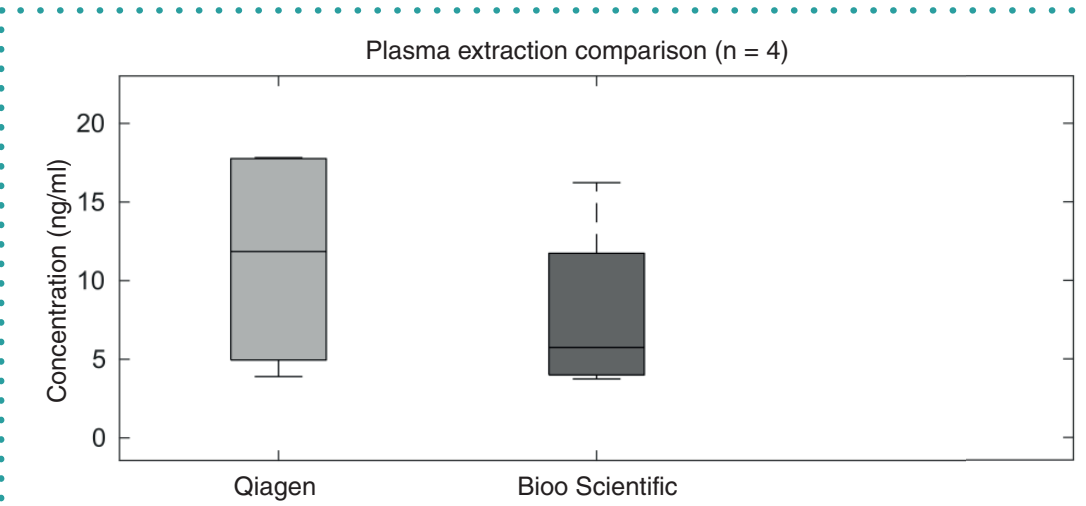

Plasma vs blood extraction $(n=81)$

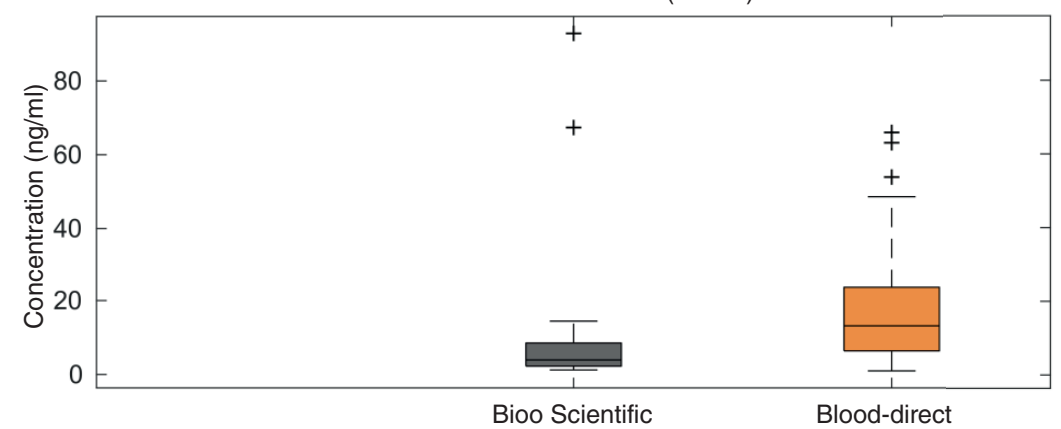

Figure 6. cfDNA extraction reproducibility. We compared the concentration $(\mathrm{ng} / \mathrm{ml})$ of DNA extracted from the plasma of four samples using the Qiagen and Bioo Scientific approaches (top). A paired 2-tail t-test comparing these concentrations did not support a significant difference $(p>0.05)$. The extraction concentration from the Bioo Scientific and blood-direct methods were compared for 81 samples (bottom) to give a $p$-value of $p=0.022$.

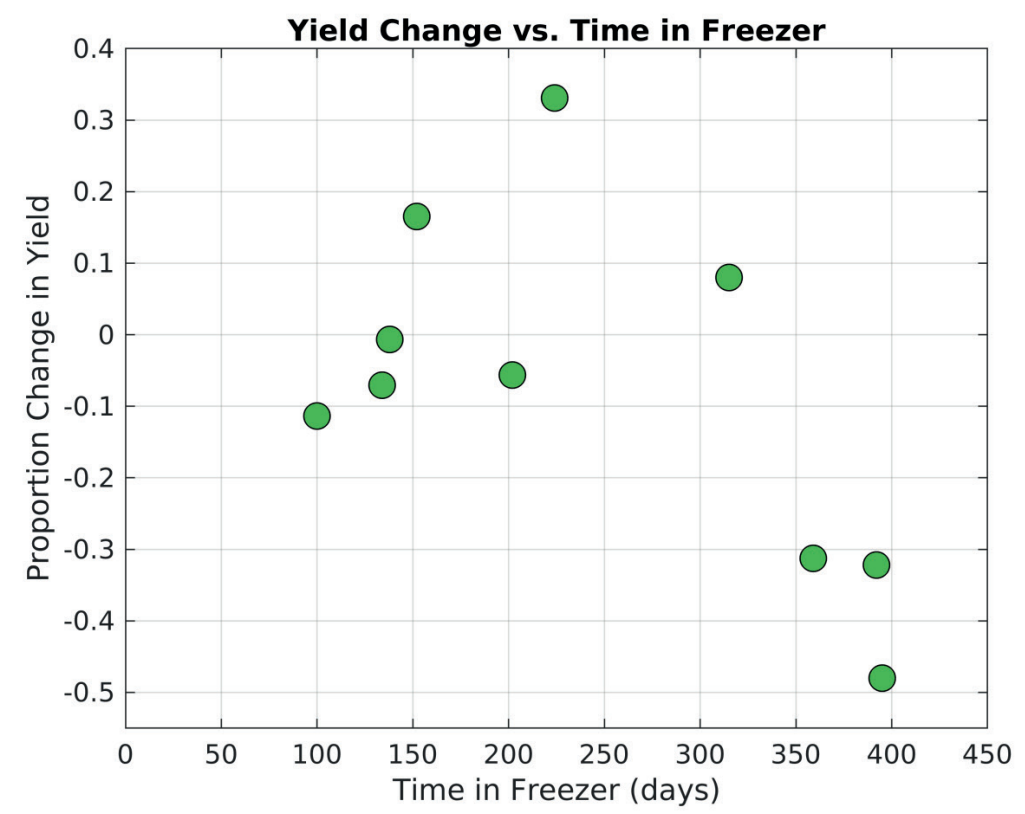

Figure 7. Yield difference versus time. We quantified the cfDNA after storing it in the freezer at $-20^{\circ} \mathrm{C}$ for 3.3-13.1 months. The correlation rho is -0.5875 with a $p$-value of 0.0741 suggesting only a weak relationship between yield difference and time in storage. 


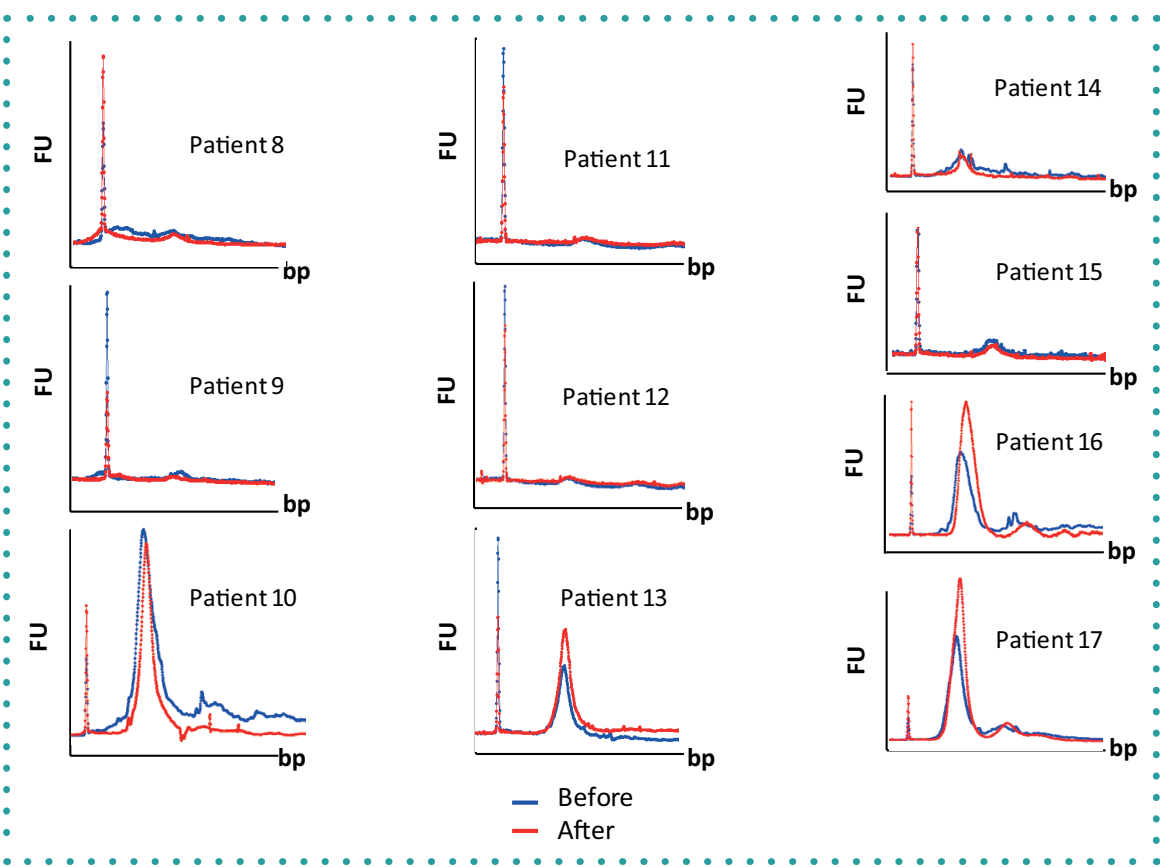

Figure 8. cfDNA stability test. 10 cancer patient's cfDNA samples were thawed and reanalyzed on Agilent Bioanalyzer $\mathrm{HS}$ assay to determine stability after storage at $-20^{\circ} \mathrm{C}$ over a period of time, ranging from 3.3 to 13.1 months. The overlays of the Agilent curves show little to no degradation between the initial and post-storage profiles. As expected, there is a lot of variability among patient cfDNA profiles, however, there is no consistent difference in cfDNA profiles caused by long-term storage. These results were further confirmed by performing a Qubit fluorometric quantification assay on the cfDNA (see Figure 7).

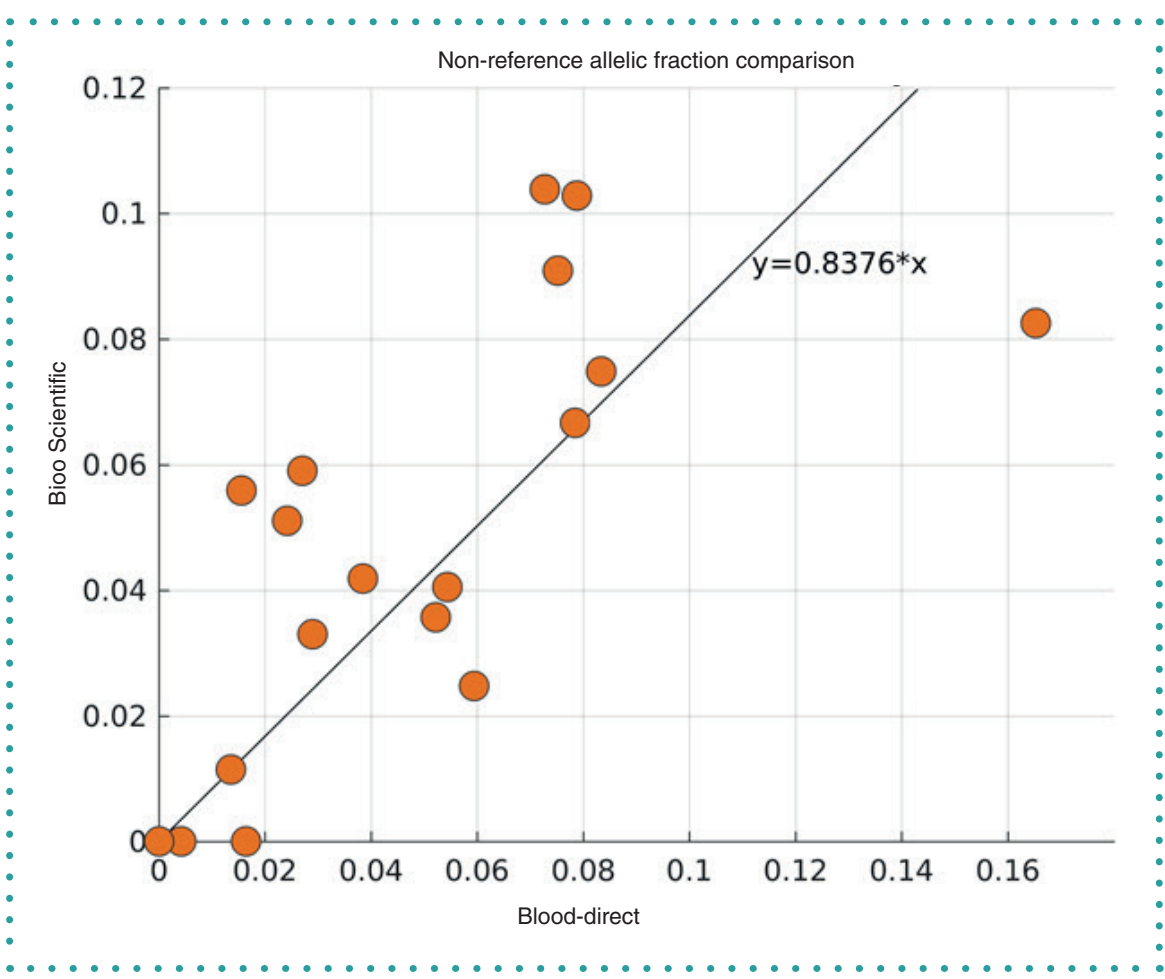

Figure 9. Whole genome-derived somatic variants were queried in the plasma and blood-direct cfDNA data to allow for comparison of non-reference allele fractions. Each point on the graph represents the allele fractions of the 19 variants. It shows that the allele fractions in the Bioo Scientific plasma method are slightly lower than those from the blood-direct method at $y=0.8376^{\star} x$. However, when the rightmost data point is removed, the data are more similar with $y=1.0485^{\star} x$. Using all data points yields a robust Pearson correlation of 0.7371 .
10. Laskin J, Jones S, Aparicio S et al. Lessons learned from the application of whole-genome analysis to the treatment of patients with advanced cancers. Cold Spring Harb. Mol. Case Stud. 1(1), a000570 (2015).

11. Jamshidi F, Pleasance E, Li Y et al. Diagnostic value of next-generation sequencing in an unusual sphenoid tumor. Oncologist 19(6), 623-630 (2014).

12. Sheffield BS, Tinker AV, Shen $Y$ et al. Personalized oncogenomics: clinical experience with malignant peritoneal mesothelioma using whole genome sequencing. PLoS One 10(3), e0119689 (2015).

13. Ronsley R, Rassekh SR, Shen Y et al. Application of genomics to identify therapeutic targets in recurrent pediatric papillary thyroid carcinoma. Cold Spring Harb. Mol. Case Stud. 4(2), a002568 (2018).

14. Alcaide M, Yu S, Davidson J et al. Targeted error-suppressed quantification of circulating tumor DNA using semi-degenerate barcoded adapters and biotinylated baits. Sci. Rep. 7(1), 10574 (2017).

15. Li H. Aligning sequence reads, clone sequences and assembly contigs with BWA-MEM. arXiv preprint arXiv:1303.3997 (2013).

16. Tarasov A, Vilella AJ, Cuppen E, Nijman IJ, Prins P. Sambamba: fast processing of NGS alignment formats. Bioinformatics 31(12), 2032-2034 (2015).

17. Saunders CT, Wong WSW, Swamy S, Becq J, Murray LJ, Cheetham RK. Strelka: accurate somatic small-varian calling from sequenced tumor-normal sample pairs. Bioinformatics 28(14), 1811-1817 (2012).

18. Fernando MR, Jiang C, Krzyzanowski GD, Ryan WL. New evidence that a large proportion of human blood plasma cell-free DNA is localized in exosomes. PLoS One 12(8), e0183915 (2017)

19. Samorodnitsky E, Datta J, Jewell BM et al. Comparison of custom capture for targeted next-generation DNA sequencing. J. Mol. Diagn. 17(1), 64-75 (2015).

20. Akane A, Matsubara $K$, Nakamura $H$, Takahashi S, Kimura K. Identification of the heme compound copurified with deoxyribonucleic acid (DNA) from bloodstains, a major inhibitor of polymerase chain reaction (PCR) amplification. J. Forensic. Sci. 39(2), 362-372 (1994).

21. Al-Harti SA. Assessment of three blood genomic-DNA preparation methods for malaria molecular diagnosis. $J$ Egypt. Soc. Parasitol. 46(1), 1-8 (2016).

22. Tack LC, Thomas M, Reich K. Automated forensic DNA purification optimized for FTA card punches and identifipurification optimized for FTA card punches and identifi$231-236(2005)$ 